\title{
The Transmuted Generalized Gamma Distribution: Properties and Application
}

\author{
Sadraque E.F. Lucena ${ }^{1}$, Ana Herm'1nia A. Silva², Gauss M. Cordeiro ${ }^{3}$ \\ Universidade Federal de Pernambuco
}

Departamento de Estat'istica, Cidade Universitaria

\begin{abstract}
The generalized gamma model has been used in several applied areas such as engineering, economics and survival analysis. We provide an extension of this model called the transmuted generalized gamma distribution, which includes as special cases some lifetime distributions. The proposed density function can be represented as a mixture of generalized gamma densities. Some mathematical properties of the new model such as the moments, generating function, mean deviations and Bonferroni and Lorenz curves are provided. We estimate the model parameters using maximum likelihood. We prove that the proposed distribution can be a competitive model in lifetime applications by means of a real data set.
\end{abstract}

Key words: maximum likelihood, mean deviation, moment generating function, generalized gamma distribution.

\section{Introduction}

Standard lifetime distributions usually present very strong restrictions to produce bathtub curves, and thus appear to be unappropriate for analyzing data with this characteristic. The three-parameter generalized gamma ("GG" for short) distribution (Stacy, 1962) includes as special models the exponential, Weibull, gamma and Rayleigh distributions, among others. It is suitable for modeling data with hazard rate function (hrf) of different forms (increasing, decreasing, bathtub and unimodal) and also useful for estimating individual hazard functions and both relative hazards and relative times (Cox, 2008). The GG distribution has been used in several research areas such as engineering, hydrology and survival analysis. Its probability density function (pdf) and cumulative distribution function (cdf) are given by (for $\mathrm{x}>0$ )

$$
g(x)=\frac{p}{\alpha \Gamma(v)}\left(\frac{x}{\alpha}\right)^{v p-1} \exp \left[-\left(\frac{x}{\alpha}\right)^{p}\right]
$$

and $\mathrm{G}(\mathrm{x})=\gamma(v,[\mathrm{x} / \mathrm{a}] \mathrm{p}) / \Gamma(v)$, respectively, where $\Gamma(v)=\mathrm{R} \infty \omega v-1 \mathrm{e}-\omega \mathrm{d} \omega$ (for $v>0)$ is the gamma function and $\gamma(\mathrm{x}, v)=\mathrm{R} \times \omega v-1 \mathrm{e}-\omega \mathrm{d} \omega$ is the incomplete gamma function. For the density function (1), $\mathrm{a}>0$ is a scale parameter and $\mathrm{p}>0$ and $v>0$ are shape parameters. The Weibull and gamma distributions are special cases of (1) when $v=1$ and $p=1$, respectively. The GG distribution approaches the log-normal distribution when $\mathrm{a}=1$ and $v \rightarrow \infty$. We denote by a random variable having density function (1).

The GG distribution includes all four more common types of the hrf: monotonically increasing and decreasing, bathtub and unimodal (Cox et al., 2007). This property is useful in reliability and survival analysis. This model has been used in several applied areas such as engineering, economics and survival analysis. Yamaguchi (1992) used it for the analysis of permanent employment in Japan, Allenby (1999) proposed a dynamic model based on it and Cox 
et al. (2007) presented a parametric survival analysis and taxonomy of its hrf. Some extensions of the GG distribution has emerged recently. For example, Pascoa et al. (2011) proposed the Kumaraswamy generalized gamma (KwGG) distribution, Ortega et al. (2011) proposed the generalized gamma geometric distribution and Cordeiro et al. (2012) defined the beta generalized gamma (BGG) distribution.

Now, we de_ne an extended form of the density function (1) (for $\mathrm{x}>0$ ) given by

$$
g \alpha, v, p(x)=\frac{|p|}{\alpha \Gamma(v)}\left(\frac{x}{\alpha}\right)^{v p-1} \exp \left[-\left(\frac{x}{\alpha}\right)^{p}\right]
$$

Where $p$ is not zero and the other parameters are positive. The cdf corresponding to (2) becomes

$$
G_{a, \nu, p}(x)=\frac{\gamma\left(\nu,[x / a]^{p}\right)}{\Gamma(\nu)} \text { for } p>0 \text { and } G_{a, \nu, p}(x)=1-\frac{\gamma\left(\nu,[x / a]^{p}\right)}{\Gamma(\nu)} \text { for } p<0 .
$$

Several continuous univariate distributions have been extensively used in the literature for modelling data in many areas such as engineering, economics, biological studies and environmental sciences. However, applied areas such as lifetime analysis, finance and insurance clearly require extended forms of these distributions. Thereby, classes of distributions have been pro- posed in the literature by extending and creating new families of continuous distributions. These extensions generalize distributions giving more flexibility by adding one or more param- eters to the baseline model. They were pioneered by Gupta et al. (1998), who proposed the exponentiated-G ("Exp-G") distribution, by raising the cdf $\mathrm{G}(\mathrm{x})$ to a positive power parameter. Many other classes can be found in the literature such as the beta generalized (BG) family of distributions proposed by Eugene et al. (2002), the Kumaraswamy (Kw-G) family of distri- butions introduced by Cordeiro and de Castro (2011) and the exponentiated generalized (EG) family defined by Cordeiro et al. (2013).

Another family of distributions arises from the general rank transmutation (GRT) defined by Shaw and Buckley (2007). Suppose we have two cdfs F (x) and G(x) with common sample space, then the GRT is defined as

$$
P_{R 12}(t)=F\left(G^{-1}(t)\right), P_{R 21}(t)=G\left(F^{-1}(t)\right) .
$$

Note that the pair in (3) takes the unit interval $[0,1]$ and under suitable assumptions are mutual inverses and satisfy PRij $(0)=0$, PRij $(1)=1$. A quadratic rank transmutation map (QRTM) is obtained by considering PR12 $(t)=t+\lambda t(1-t)$. Then, it follows that the cdfs are related by

$$
F(x)=(1+\lambda) G(x)-\lambda G(x)^{2}, \quad|\lambda| \leq 1,
$$

and the corresponding pdf is given by

$$
f(x)=g(x)[1+\lambda-2 \lambda G(x)]
$$

Here, $\mathrm{G}(\mathrm{x})$ and $\mathrm{g}(\mathrm{x})$ can be understood as the cdf and pdf of the baseline distribution, respectively. Note that this generator, called the transmuted class (TC) of distributions, is a linear combination of 
the baseline and Exp-G distributions with power parameter equal to two. Also, note that $\lambda=0$ in (5) gives the baseline distribution. Further details can be seen in Shaw and Buckley (2007).

Some distributions belonging to the TC class have been proposed recently. Aryal and Tsokos (2009) studied the transmuted Gumbel distribution and its application to climate data. Aryal and Tsokos (2011) pioneered the transmuted Weibull distribution and used it for modelling the tensile fatigue characteristics of a polyester/viscose yarn. Khan and King (2013) intro- duced the transmuted modified Weibull distribution and Ashour and Eltehiwy (2013) defined the transmuted exponentiated Lomax model. In the present study, we provide some mathe- matical properties of a new lifetime model named the transmuted generalized gamma (TGG) distribution.

The sections are organized as follow. In Section 2, we define the TGG model. Some of its mathematical properties are investigated in Section 3. An application to a real data set is reported in Section 4. Section 5 ends with some conclusions. 


\section{The TGG distribution}

The cdf of the TGG distribution can be obtained from (4) as

$$
F(x)= \begin{cases}\frac{(1+\lambda) \gamma\left[\nu,(x / a)^{p}\right]}{\Gamma(\nu)}-\frac{\lambda \gamma\left[\nu,(x / a)^{p}\right]^{2}}{\Gamma(\nu)^{2}}, & \text { if } p>0, \\ 1+\lambda-\frac{(1+\lambda) \gamma\left[\nu,(x / a)^{p}\right]}{\Gamma(\nu)}-\lambda\left(1-\frac{\gamma\left[\nu,(x / a)^{p}\right.}{\Gamma(\nu)}\right)^{2}, & \text { if } p<0,\end{cases}
$$

where $|\lambda| \leq 1, a>0, v>0$ and $p=0$. Henceforth, a random variable $X$ having the $\operatorname{cdf}(6)$ is denoted by $\mathrm{X} \sim \mathrm{T} \operatorname{GG}(\lambda, \mathrm{a}, v, \mathrm{p})$. We can prove that the TGG distribution is a linear combination of the GG and the exponentiated generalized gamma (EGG) distributions, the last one with power parameter two. The pdf of $\mathrm{X}$ is given by

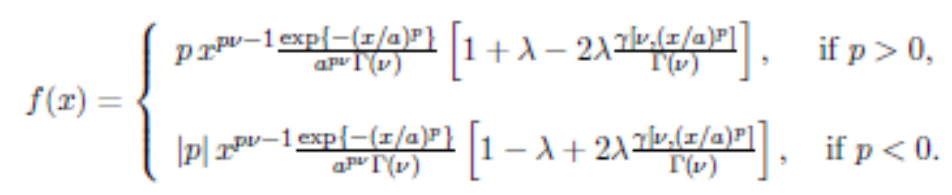

The TGG family model has many distributions as special cases. Some of the sub-models encompassed by the TGG family are listed in Table 1.

The hazard rate function (hrf) of $\mathrm{X}$ is given by

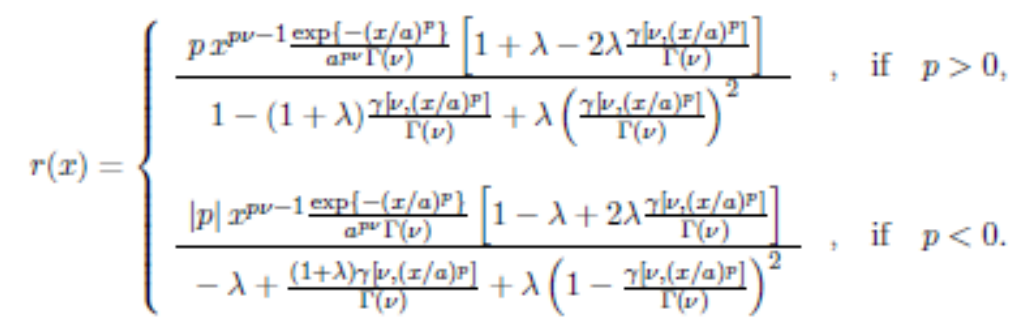

Some possible shapes of the density function (7) and the hrf are plotted in Figure 1. We conclude that the $h r f(x)$ is very flexible, assuming different shapes.

\section{Properties of the TGG distribution}

Let $\mathrm{g}(\mathrm{x})$ and $\mathrm{G}(\mathrm{x})$ are the pdf and cdf of a baseline model. A random variable is Exp-G distributed with power parameter $\alpha>0$ if its cdf and pdf are given by $\mathrm{H} \alpha(\mathrm{x})=\mathrm{G}(\mathrm{x}) \alpha$ and $\mathrm{h} \alpha(\mathrm{x})=$ $\alpha \mathrm{g}(\mathrm{x}) \mathrm{G}(\mathrm{x}) \alpha-1$, respectively. We obtain some properties of the TGG distribution. If the baseline is taken to be the GG distribution, the random variable $\mathrm{Y}$ is said to be Exp- GG distributed, say Y $\operatorname{Exp}-\mathrm{GG}(\alpha, \mathrm{a}, v, \mathrm{p})$ distribution. The density function of $\mathrm{X}$ can be expressed as

$$
f(x)=(1+\lambda) g(x)-\lambda h_{2}(x),
$$


Table 1: Special cases of the TGG family.

\begin{tabular}{lccccll}
\hline Distribution & $\lambda$ & $a$ & $p$ & $\nu$ & References \\
\hline Transmuted Weibull & $\lambda$ & $a$ & $>0$ & 1 & Aryal and Tsokos (2011) \\
Transmuted Exponential & $\lambda$ & $a$ & 1 & 1 & \\
Transmuted Gamma & $\lambda$ & $a$ & 1 & $\nu$ & \\
Transmuted Chi-squared & $\lambda$ & 2 & 1 & $q / 2$ & \\
Transmuted Chi & $\lambda$ & $\sqrt{2}$ & 2 & $q / 2$ & \\
Transmuted Rayleigh & $\lambda$ & $\sigma \sqrt{2}$ & 2 & 1 & Merovci (2013a) \\
Transmuted Reciprocal Weibull & $\lambda$ & $a$ & $<0$ & $\nu$ & \\
Transmuted Reciprocal Exponential & $\lambda$ & $a$ & -1 & 1 & \\
Transmuted Reciprocal Gamma & $\lambda$ & $a$ & -1 & $\nu$ & \\
Transmuted Reciprocal Rayleigh & $\lambda$ & $a$ & -2 & 1 & \\
Transmuted Reciprocal Chi-squared & $\lambda$ & $1 / 2$ & -1 & $q / 2$ & \\
Transmuted Reciprocal Chi & $\lambda$ & $\sqrt{2}$ & -2 & $q / 2$ & \\
Transmuted Scaled Reciprocal-Chi & $\lambda$ & $\sigma \sqrt{2}$ & -2 & $q / 2$ & \\
Generalized Gamma & 0 & $a$ & $p$ & $\nu$ & \\
Weibull & 0 & $a$ & $>0$ & 1 & \\
Exponential & 0 & $a$ & 1 & 1 & \\
Gamma & 0 & $a$ & 1 & $\nu$ & \\
Chi-squared & 0 & 2 & 1 & $q / 2$ & \\
Chi & 0 & $\sqrt{2}$ & 2 & $q / 2$ & \\
Rayleigh & 0 & $\sigma \sqrt{2}$ & 2 & 1 & \\
Reciprocal Weibull & 0 & $a$ & $<0$ & $\nu$ & \\
Reciprocal Exponential & 0 & $a$ & -1 & 1 & \\
Reciprocal Gamma & 0 & $a$ & -1 & $\nu$ & \\
Reciprocal Rayleigh & 0 & $a$ & -2 & 1 & \\
Reciprocal Chi-squared & 0 & $1 / 2$ & -1 & $q / 2$ & \\
Reciprocal Chi & 0 & $\sqrt{2}$ & -2 & $q / 2$ & \\
Scaled Reciprocal-Chi & 0 & $\sigma \sqrt{2}$ & -2 & $q / 2$ & \\
\hline & & & & & \\
& & & &
\end{tabular}

where $\mathrm{g}(\mathrm{x})$ is the GG pdf given by (2) and $\mathrm{h} 2(\mathrm{x})=2 \mathrm{~g}(\mathrm{x}) \mathrm{G}(\mathrm{x})$ is the pdf of the $\operatorname{Exp}-\mathrm{GG}(2, \mathrm{a}, \mathrm{v}, \mathrm{p})$ model. The pdf of the Exp-GG(2, a, $v, p)$ distributed is given by

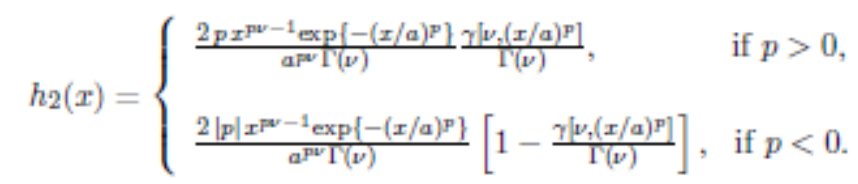

Equation (8) reveals that the TGG pdf is a linear combination of the GG and Exp-GG densities, the last one with power parameter two. Using the power series

$$
\frac{\gamma\left[\nu,(x / a)^{p}\right]}{\Gamma(\nu)}=\frac{1}{\Gamma(\nu)} \sum_{i=0}^{\infty} \frac{(-1)^{i} x^{p(\nu+i)}}{a^{p(\nu+i)}(\nu+i) i !},
$$

the Exp-GG(2, a, v, p) density function becomes

$$
h(x)= \begin{cases}\sum_{i=0}^{\infty} w_{i} g_{a, V_{i}^{*}, p}(x), & \text { if } p>0, \\ 2 g_{a, \nu, p}(x)-\sum_{i=0}^{\infty} w_{i} g_{a, \nu_{i}^{*}, p}(x), & \text { if } p<0,\end{cases}
$$

where ga, $v i *, p(x)$ denotes the GG density function with parameters a, vi and $\mathrm{p}, v \mathrm{i}=2 v+\mathrm{i}$ and wi $=2(-1) i \Gamma(2 v+\mathrm{i}) /[(v+\mathrm{i}) \mathrm{i} ! \Gamma(v) 2]$. Then, the pdf of $\mathrm{X}$ can be expressed as

$$
f(x)= \begin{cases}(1+\lambda) g_{a, \nu, p}(x)-\lambda \sum_{i=0}^{\infty} w_{i} g_{a, V_{i}^{*}, p}(x), & \text { if } p>0, \\ (1-\lambda) g_{a, \nu, p}(x)+\lambda \sum_{i=0}^{\infty} w_{i} g_{a, V_{i}^{*}, p}(x), & \text { if } p<0 .\end{cases}
$$



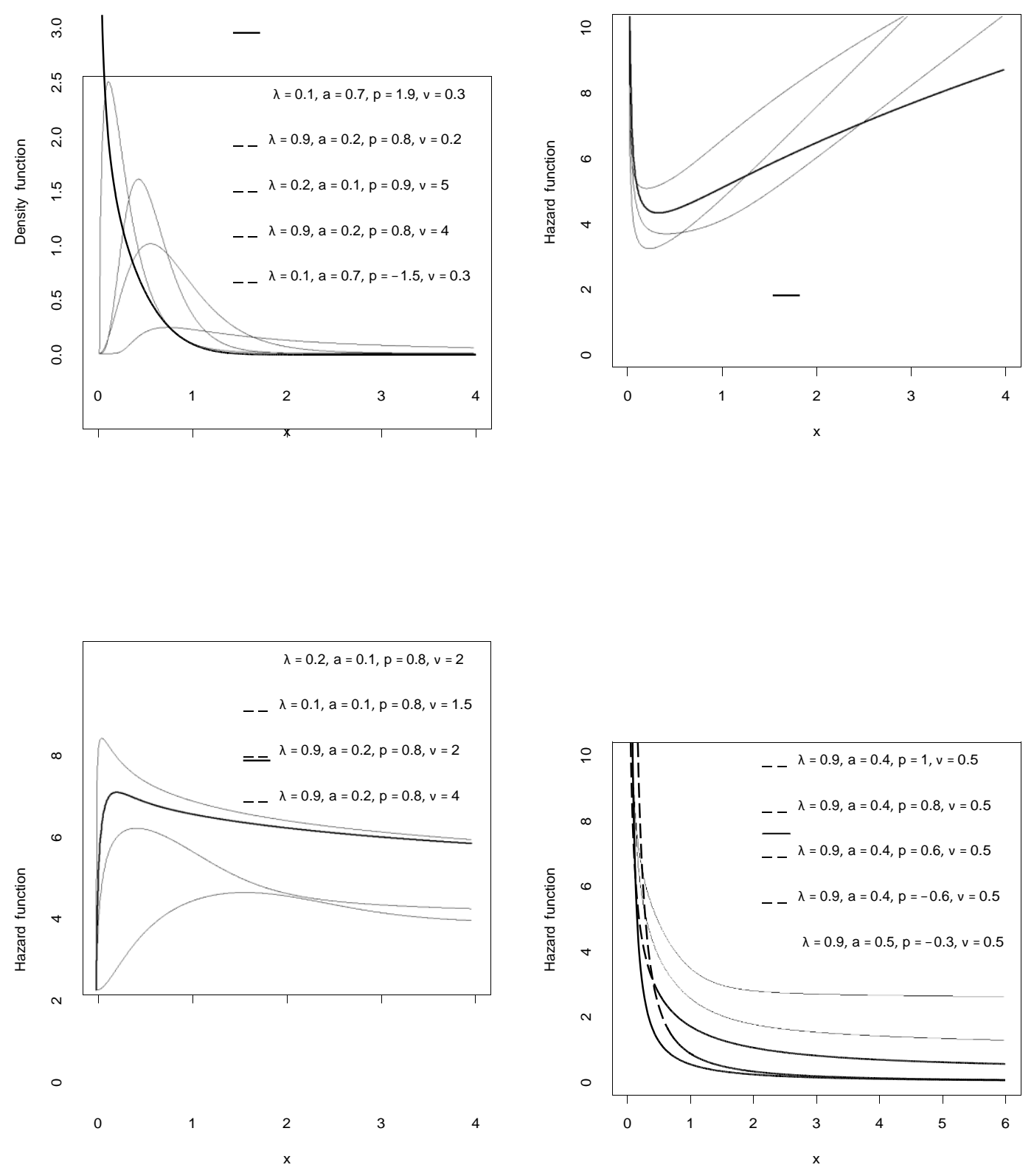

Figure 1: Plots of the TGG pdf and hrf for some parameter values

Equation (9) reveals that the TGG distribution is a mixture of GG distributions. This result enable us to derive some mathematical properties of the TGG distribution.

\subsection{Moments}

Some of the most important features and characteristics of a distribution can be studied through moments like tendency, dispersion, skewness and kurtosis. For a random variable $Z$ having the $G G(a$, $v, p)$ distribution, the sth moment of $Z$ becomes $\operatorname{IE}(Z s)=$ as $\Gamma(v+s / p) / \Gamma(v)$. Based on equation (9), the sth moment of $X$ is given by 


$$
\mathbb{E}\left(X^{s}\right)=\left\{\begin{array}{cl}
(1+\lambda) \frac{a^{x} \Gamma(\nu+s / p)}{\Gamma(\nu)}-\lambda a^{s} \sum_{i=0}^{\infty} w_{i} \frac{\Gamma\left(\nu_{i}^{*}+s / p\right)}{\Gamma\left(\nu^{*}\right)}, & \text { if } p>0, \\
(1-\lambda) \frac{a^{\alpha} \Gamma(\nu+s / p)}{\Gamma(\nu)}+\lambda a^{s} \sum_{i=0}^{\infty} w_{i} \frac{\Gamma\left(\nu_{i}^{*}+s / p\right)}{\Gamma\left(\nu^{*}\right)}, & \text { if } p<0 .
\end{array}\right.
$$

These moments can be computed numerically by standard statistical softwares.

\subsection{Generating Function}

The moment generating function (mgf) of $Z$, say Ma,v,p $(s)=I E(e s Z)$, has an explicit expression using the Wright function (Wright, 1935). It can be expressed as

$$
M_{a, \nu, p}(s)=\frac{|p|}{a \Gamma(\nu)} \int_{0}^{\infty} \exp (s x)(x / a)^{p \nu-1} \exp \left[-(x / a)^{p}\right] d x .
$$

Setting $\mathrm{u}=\mathrm{x} / \mathrm{a}$, we have

$$
M_{a, \nu, p}(s)=\frac{|p|}{\Gamma(\nu)} \int_{0}^{\infty} \exp (a s u) u^{p \nu-1} \exp \left(-u^{p}\right) d u .
$$

By expanding the first exponential in power series and the result $\int_{0}^{\infty} u^{p v-1} \exp \left(-u^{p}\right) d u=$ $p^{-1} \Gamma(v+m / p)$, we obtain

$$
M_{a, \nu, p}(s)=\frac{\operatorname{sgn}(p)}{\Gamma(\nu)} \sum_{m=0}^{\infty} \Gamma\left(\nu+\frac{m}{p}\right) \frac{(a s)^{m}}{m !} .
$$

The above equation holds for $p=0$. Additionally, for a given $p>1$, it can be expressed in terms of the Wright generalized hypergeometric function (Wright, 1935) defined by

$$
{ }_{p} \Psi_{q}\left[\begin{array}{l}
\left(\alpha_{1}, A_{1}\right), \ldots,\left(\alpha_{p}, A_{p}\right) \\
\left(\beta_{1}, B_{1}\right), \ldots,\left(\beta_{q}, B_{q}\right)
\end{array}\right]=\sum_{m=0}^{\infty} \frac{\prod_{j=1}^{p} \Gamma\left(\alpha_{j}+A_{j} m\right)}{\prod_{j=1}^{q} \Gamma\left(\beta_{j}+B_{j} m\right)} \frac{x^{m}}{m !}
$$

This function exists if $1+\sum_{j=1}^{q} B_{j}-\sum_{j=1}^{q} A_{j}>0$. By combining the last two equations, we obtain

$$
M_{a, \nu, p}(s)=\frac{1}{\Gamma(\nu)}{ }_{1} \Psi_{0}\left\lfloor\begin{array}{c}
\left(\nu, p^{-1}\right) \\
-
\end{array} ; \text { as }\right\rfloor .
$$

Then, from equations (9) and (10), the mgf of $X$ reduces to

$$
M(s)= \begin{cases}(1+\lambda) M_{a, \nu, p}(s)-\lambda \sum_{i=0}^{\infty} w_{i} M_{a, \nu^{*}, p}(s), & \text { if } p>0, \\ (1-\lambda) M_{a, \nu, p}(s)+\lambda \sum_{i=0}^{\infty} w_{i} M_{a, \nu^{*}, p}(s), & \text { if } p<0,\end{cases}
$$

where $M a, v *, p(x)$ is the mgf of $Z$ with parameters $a, v *$ and $p$. This result reveals that the TGG mgf is a mixture of GG mgfs. 


\subsection{Mean Deviations}

One way to measure the amount of scatter in a population is the totality of deviations about the mean and the median. The mean deviation of $X$ about the mean $\mu=E(X)$ and about the median $M$ are defined as $\delta 1=R \infty|x-\mu| f(x) d x$ and $\delta 2=R \infty|x-M| f(x) d x$, respectively. They can also be expressed as $\delta 1=2 \mu \mathrm{F}(\mu)-2 \mathrm{~m} 1(\mu)$ and $\delta 2=\mu-2 \mathrm{~m} 1(\mathrm{M})$, where $F(\mu)$ is calculated from (6) and $m 1(u)=R u x f(x) d x$. From equation (9), we can write

$$
m_{1}(u)= \begin{cases}\frac{a(1+\lambda) \Gamma(\nu+1 / p)}{\Gamma(\nu)} G_{a, \nu+1 / p, p}(u)-\lambda a \sum_{i=0}^{\infty} w_{i} \frac{\Gamma\left(\nu^{*}+1 / p\right)}{\Gamma\left(\nu^{*}\right)} G_{a, \nu^{*}+1 / p, p}(u), & \text { if } p>0, \\ \frac{a(1-\lambda) \Gamma(\nu+1 / p)}{\Gamma(\nu)} G_{a, \nu+1 / p, p}(u)+\lambda a \sum_{i=0}^{\infty} w_{i} \frac{\Gamma\left(\nu^{*}+1 / p\right)}{\Gamma\left(\nu^{*}\right)} G_{a, \nu^{*}+1 / p, p}(u), & \text { if } p<0 .\end{cases}
$$

Equation (11) can also be used to obtain Bonferroni and Lorenz curves, which are useful in many fields like reliability, demography, economics, insurance and medicine. For the TGG distribution, these curves can be calculated (for given $0<\pi<1$ ) from $B(\pi)=(\pi \mu)-1 \mathrm{~m} 1$ (q) and $L(\pi)=\mu-1 \mathrm{~m} 1(\mathrm{q})$, respectively, where $q=F-1(\pi)$ is the quantile function (qf) at $\pi$. The Bonferroni and Lorenz curves for some values of the parameters are displayed in Figure 2. 

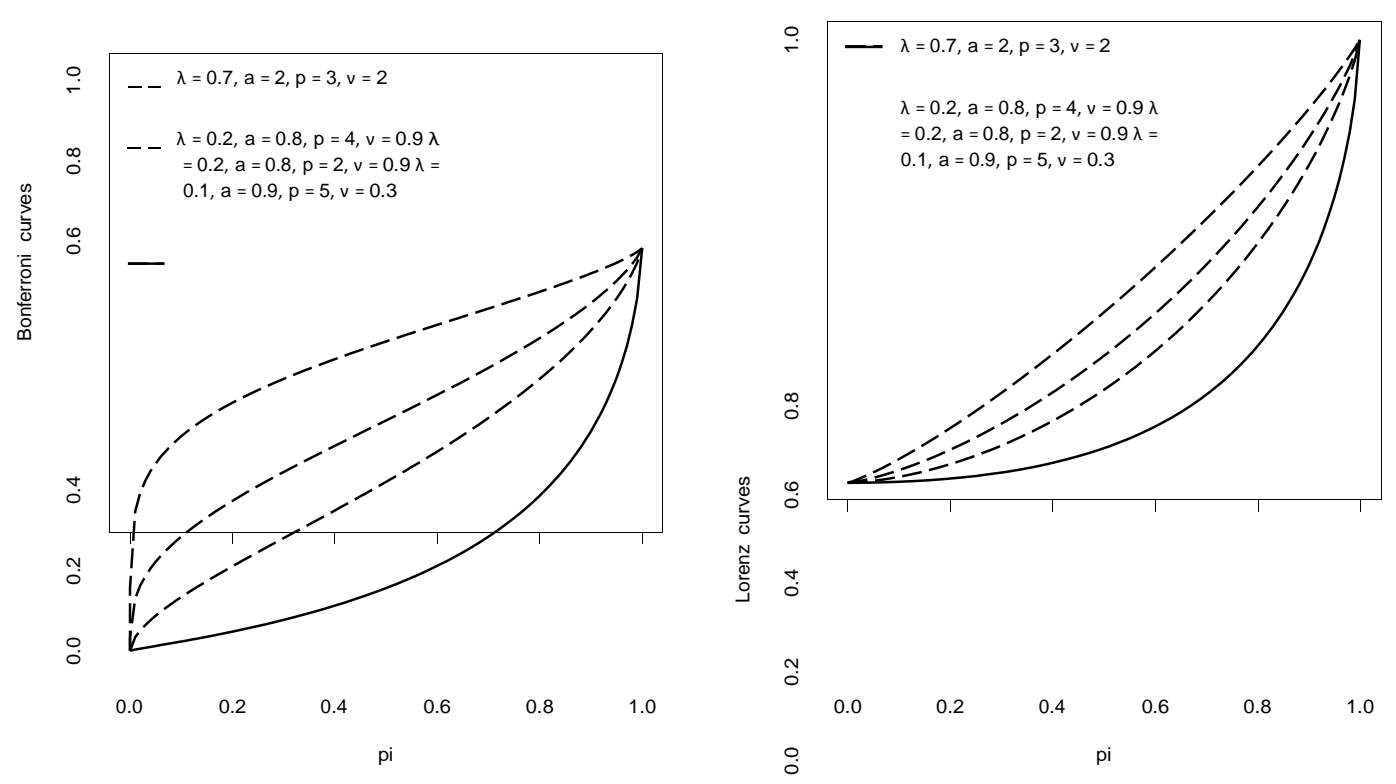

Figure 2: Bonferroni and Lorenz curves for some parameter values.

\subsection{Skewness and Kurtosis}

The qf of $X$, say $x=Q(u)$, follows by inverting the cdf (6). It is given by

$$
x=Q(u)= \begin{cases}Q_{G G}\left(\frac{\left[1+\lambda-\sqrt{(1+\lambda)^{2}-4 \lambda u}\right]}{2 \lambda}\right), & \text { if } p>0, \\ Q_{G G}\left(1-\frac{\left[1+\lambda-\sqrt{(1+\lambda)^{2}-4 \lambda u}\right]}{2 \lambda}\right), & \text { if } p<0,\end{cases}
$$

where QGG denotes the $q$ f of the GG distribution with parameters $a, v$ and $p$.

There are several robust measures in the literature for location and dispersion. The median, for example, can be used for location and the interquartile range. Both the median and the interquartile range are based on quantiles. From this fact, Bowley (1920) proposed a coefficient of skewness based on quantiles given by

$$
S K=\frac{Q(3 / 4)+Q(1 / 4)-2 Q(1 / 2)}{Q(3 / 4)-Q(1 / 4)},
$$

where $Q(\cdot)$ here is the qf of the TGG distribution given by (12). Moors (1988) demonstrated that the conventional measure of kurtosis may be interpreted as a dispersion around the values $\mu+\sigma$ and $\mu-\sigma$, where $\mu$ is the mean of the distribution and $\sigma$ is its standard error. Thus, the probability mass focuses around $\mu$ or on the tails of the distribution. Therefore, based on this

$$
K R=\frac{[Q(7 / 8)-Q(5 / 8)]+[Q(3 / 8)-Q(1 / 8)]}{Q(6 / 8)-Q(2 / 8)} .
$$


interpretation, Moors (1988) proposed, as an alternative to the conventional coefficient of kurtosis, a robust measure based on octiles given by

These measures are less sensitive to outliers and they exist even for distributions without moments. Figure 3 displays the plots of the Bowleys skewness and Moors kurtosis for the TGG distribution, respectively.

\subsection{Estimation}

The parameters of the TGG distribution can be estimated by the method of maximum lakelihood. Let $x 1, \ldots, x n$ be a sample of size $n$ from the $\operatorname{TGG}(\lambda, a, v, p)$ distribution given by 
$\stackrel{\bullet}{\circ}$

$\stackrel{\circ}{\circ}$

¿̊.
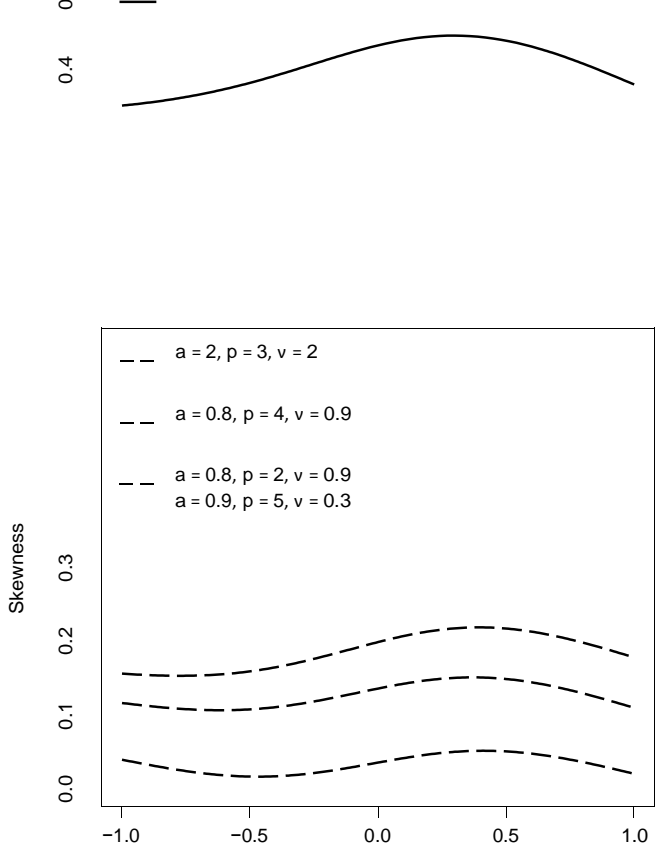

$\lambda$
이

$\stackrel{\infty}{\sim}$

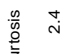

$\stackrel{\text { ก }}{\mathfrak{n}}$

$\stackrel{\circ}{\mathrm{i}}$

$\stackrel{\infty}{-}$

$\begin{array}{lllll}-1.0 & -0.5 & 0.0 & 0.5 & 1.0\end{array}$

Figure 3: Skewness and Kurtosis of the TGG distribution for different values of $\lambda$.

(7). Let $\theta=(\lambda, a, v, p)^{>}$be the vector of parameters. The log-likelihood function for $\theta$ can be expressed as

$$
\begin{aligned}
\ell(\theta)= & n \log |p|-n p \nu \log (a)-n \log \Gamma(\nu)+(p \nu-1) \sum_{i=0}^{n} \log \left(x_{i}\right)-\sum_{i=0}^{n}\left(\frac{x}{a}\right)^{p} \\
& +\sum_{i=0}^{n} \log \left(1+\lambda-2 \lambda \frac{\gamma\left[\nu,\left(x_{i} / a\right)^{p}\right]}{\Gamma(\nu)}\right) .
\end{aligned}
$$




$$
\begin{aligned}
& U_{\lambda}(\theta)=\frac{\partial \ell}{\partial \lambda}=\sum_{i=0}^{n} \frac{2 \gamma\left[\nu,\left(x_{i} / a\right)^{p}\right]-\Gamma(\nu)}{2 \gamma\left[\nu,\left(x_{i} / a\right)^{p}\right]-(1+\lambda) \Gamma(\nu)}, \\
& U_{a}(\theta)=\frac{\partial \ell}{\partial a}=-\frac{n p \nu}{a}+\frac{p}{a^{p+1}} \sum_{i=0}^{n} x_{i}^{p}+\sum_{i=0}^{n} \frac{2 \lambda \frac{\partial \gamma\left[\nu,\left(x_{i} / a\right) \mathrm{P}\right]}{\partial a}}{2 \lambda \gamma\left[\nu,\left(x_{i} / a\right)^{p}\right]-(1+\lambda) \Gamma(\nu)}, \\
& U_{\nu}(\theta)=\frac{\partial \ell}{\partial \nu}=-n p \log (a)-n \psi(\nu)+p \sum_{i=1}^{n} \log \left(x_{i}\right)+\sum_{i=1}^{n} \frac{2 \lambda\left\{\frac{\partial \gamma\left[\nu,\left(x_{i} / a\right) \mathrm{P}\right]}{\partial \nu}-\psi(\nu) \gamma\left[\nu,\left(x_{i} / a\right)^{p}\right]\right\}}{2 \lambda \gamma\left[\nu,\left(x_{i} / a\right)^{p}\right]-(1+\lambda) \Gamma(\nu)}, \\
& U_{p}(\theta)=\frac{\partial \ell}{\partial p}=\frac{n}{|p|}-n \nu \log (a)+\nu \sum_{i=1}^{n} \log \left(x_{i}\right)+\sum_{i=1}^{n}\left(\frac{x_{i}}{a}\right)^{p} \log \left(\frac{x_{i}}{a}\right) \\
& +\sum_{i=1}^{n} \frac{2 \lambda \frac{\partial \gamma\left[\nu,\left(x_{i} / a\right) \mathrm{P}\right]}{\partial_{p}}}{2 \lambda \gamma\left[\nu,\left(x_{i} / a\right)^{p}\right]-(1+\lambda) \Gamma(\nu)} .
\end{aligned}
$$

The components of the score vector $U(\theta)$ are given by

These expressions depend on the quantities $\partial \gamma(\cdot) / \partial a, \partial \gamma(\cdot) / \partial p$ and $\partial \gamma(\cdot) / \partial v$. Now, we provide formulae for these quantities. The first and the second derivatives are obtained using MATHEMATICA as

and

$$
\frac{\partial \gamma\left[\nu,\left(x_{i} / a\right)^{p}\right]}{\partial a}=-\frac{p x^{p \nu} \exp \left[-\left(x_{i} / a\right)^{p}\right]}{a^{p+1}}
$$

$$
\frac{\partial \gamma\left[\nu,\left(x_{i} / a\right)^{p}\right]}{\partial p}=\left(\frac{x_{i}}{a}\right)^{p \nu} \log \left(\frac{x_{i}}{a}\right) \exp \left[-\left(x_{i} / a\right)^{p}\right]
$$

respectively. Further, these are two di_erent forms to calculate $\frac{\theta \gamma}{\theta p}\left[v,\left(x_{i} / \alpha\right)^{p}\right]$. The first one is

$$
\frac{\partial \gamma\left[\nu,\left(x_{i} / a\right)^{p}\right]}{\partial \nu}=\Gamma(\nu) \psi(\nu)-\log \left[(x / a)^{P}\right] \gamma\left[\nu,\left(x_{i} / a\right)^{p}\right]-G_{23}^{30}\left[\left.(x / a)^{p}\right|_{0,0, \nu} ^{1,1}\right],
$$

where $\psi(\cdot)$ is the digamma function and $G_{23}^{30}$ is a special case of the Meijer G-function given by $G_{23}^{30}\left[\left.(x / a)^{p}\right|_{0,0, \nu} ^{1,1}\right]=\frac{(x / a)^{p \nu}}{{ }_{2} F_{2}\left(\{\nu, \nu\} ;\{\nu+1, \nu+1\} ;-(x / a)^{p}\right)} \nu^{2}-\Gamma(\nu) \log \left[(x / a)^{p}\right]+\Gamma(\nu) \psi(\nu)$

and 2 F2 $(\because ; \cdot \cdot)$ denotes the hypergeometric function defined by

$$
{ }_{2} F_{2}\left(\left\{a_{1}, a_{2}\right\} ;\left\{b_{1}, b_{2}\right\} ; z\right)=\sum_{j=0}^{\infty} \frac{\left(a_{1}\right)_{j}\left(a_{2}\right)_{j}}{\left(b_{1}\right)_{j}\left(b_{2}\right)_{j}} \frac{z^{j}}{j !}
$$


where, for some parameter $\xi$, the Pochhammer symbol $(\xi)$ j is defined by

$$
(\xi)_{0}=1, \quad(\xi)_{j}=\xi(\xi+1) \ldots(\xi+j-1), j=1,2, \ldots
$$

The last one is given by

$$
\frac{\partial \gamma\left[\nu,\left(x_{i} / a\right)^{p}\right]}{\partial \nu}=\log \left[(x / a)^{p}\right]\left\{\Gamma(\nu)-\gamma\left[\nu,(x / a)^{p}\right]\right\}-\frac{(x / a)^{p}}{{ }_{2} F_{2}\left(\{\nu, \nu\} ;\{\nu+1, \nu+1\} ;-(x / a)^{p}\right)} \nu^{2} .
$$

The maximum likelihood estimate (MLE) $\theta b$ of $\theta$ is the solution of the system of nonlinear equations $U(\theta)=0$. For interval estimation and hypothesis tests on the parameters in $\theta$, we require the $4 \times 4$ unit observed information matrix $J=J(\theta)$, whose elements are given in the Appendix. Under certain regularity conditions, they can be constructed using the fact that the asymptotic distribution of the MLE $\theta b$ is $(\theta b-\theta) \sim N p(0, I(\theta)-1)$, where I $(\theta)$ is the expected information matrix and $p$ is the number of model parameters (Sen and Singer, 1993). We can substitute $I(\theta)$ by $J(\theta b)$, i.e., the observed information matrix evaluated at $\theta b$. The multivariate normal $\mathrm{N} 5(0, \mathrm{~J}(\theta \mathrm{b})-1)$ distribution can be used to obtain approximated confidence intervals for the individual parameters. We can compute the maximum values of the unrestricted and restricted log-likelihoods to define likelihood ratio (LR) statistics for testing some sub-models of the TGG distribution.

\section{Application}

We use a real data set to show that the TGG distribution provide a better fit than that one based on the GG distribution. We also emphasize that the fitted TGG distribution is better than the fitted EGG (Cordeiro et al., 2011), BGG (Cordeiro et al., 2012) and Marshall-Olkin generalized gamma (MOGG) distributions to these data. The corresponding cdf's are given by

$$
\begin{gathered}
F_{E G G}(x)=G_{a, \nu, p}(x)^{\lambda}, \quad F_{B G G}(x)=\frac{1}{B(\alpha, \lambda)} \int_{0}^{G_{a, \nu, P}(x)} \omega^{\alpha-1}(1-\omega)^{\lambda-1} d \omega \\
\text { and } F_{M O G G}(x)=1-\frac{\lambda \bar{G}_{a, \nu, p}(x)}{G_{a, \nu, p}(x)-\lambda \bar{G}_{a, \nu, p}(x)},
\end{gathered}
$$

where $\alpha>0, \lambda>0, \mathrm{Ga}, v, \mathrm{p}$ (x) denotes the GG cdf and $\bar{G}_{a, v, p}(x)$ is the survival function.

The data represent the times between successive failures (in thousands of hours) in events of secondary reactor pumps studied by Salman et al. (1999). Table 2 gives some statistic measures for these data, which indicate that the empirical distribution is skewed to the left and platycurtic.

Table 2: Descriptive statistics for the times between successive failures (in thousands of hours) of secondary reactor pumps

$\begin{array}{lrrrrrrr}\text { Mean } & \text { Median } & \text { Std. Dev. } & \text { Variance } & \text { Skewness } & \text { Kurtosis } & \text { Min } & \text { Max } \\ 1.5779 & 0.6140 & 1.9307 & 3.7275 & 1.3643 & 3.5445 & 0.0620 & 6.5600\end{array}$


For the purpose of comparing the fits of the BGG, TGG, EGG, MOGG and GG distributions, the maximum likelihood estimation method was adopted in the R software using the subroutine

'fitdistr' (from the MASS library). The MLEs, their standard errors and the statistics AIC (Akaike Information Criterion), BIC (Bayesian Information Criterion) and CAIC (Consistent Akaike Information Criterion) are reported in Table 3. As one may note, the lowest values of the statistics correspond to the TGG distribution. The LR statistic is also calculated to test H0: GG versus H1: T GG, yielding $\Lambda=4.2240$ (1 d.f., $p$-value $=0.0399$ ). Therefore, we reject the null hypothesis in favor of the TGG distribution at the $5 \%$ level of significance. The plots of the fitted densities to the data are displayed in Figure 4. Since the EGG and the GG plots are very similar, the first one was omitted. Note that the BGG, TGG and MOGG models are similar according to the graphical comparison and that the GG model yields the worse fit. Based on the AIC and BIC statistics and the plots, we can conclude that the TGG distribution gives a better fit than the other models.

Table 3: MLEs and Goodness-of-_t measures

\begin{tabular}{|c|c|c|c|c|c|c|c|c|}
\hline \multirow[t]{2}{*}{ Model } & \multicolumn{5}{|c|}{$\begin{array}{c}\text { Estimates } \\
\text { (standard errors) }\end{array}$} & \multicolumn{3}{|c|}{$\begin{array}{l}\text { Goodness-of-fit } \\
\text { measures }\end{array}$} \\
\hline & $\widehat{\alpha}$ & $\lambda$ & $\widehat{a}$ & $\hat{v}$ & $\widehat{p}$ & $\mathrm{AIC}$ & $\mathrm{BIC}$ & CAIC \\
\hline BGG & $\begin{array}{c}8.8628 \\
(21.8536)\end{array}$ & $\begin{array}{c}64.9737 \\
(282.5036)\end{array}$ & $\begin{array}{c}0.0557 \\
(0.3627)\end{array}$ & $\begin{array}{c}3.1966 \\
(1.2240)\end{array}$ & $\begin{array}{c}0.1037 \\
(0.0841)\end{array}$ & 73.6501 & 79.3276 & 76.4889 \\
\hline TGG & - & $\begin{array}{c}0.1873 \\
(0.6511)\end{array}$ & $\begin{array}{c}0.0228 \\
(0.0795)\end{array}$ & $\begin{array}{c}4.2443 \\
(3.0245)\end{array}$ & $\begin{array}{c}0.3706 \\
(0.1440)\end{array}$ & 71.8127 & 76.3547 & 74.0837 \\
\hline Exp-GG & - & $\begin{array}{c}5.2578 \\
(23.6515)\end{array}$ & $\begin{array}{c}6.7983 \\
(0.4010)\end{array}$ & $\begin{array}{c}0.0039 \\
(0.0020)\end{array}$ & $\begin{array}{c}21.7510 \\
(97.2044)\end{array}$ & 76.0237 & 80.5657 & 78.2947 \\
\hline MOGG & - & $\begin{array}{c}65.2855 \\
(273.7025)\end{array}$ & $\begin{array}{c}0.0127 \\
(0.0249)\end{array}$ & $\begin{array}{c}0.7180 \\
(1.7895)\end{array}$ & $\begin{array}{c}0.3042 \\
(0.0858)\end{array}$ & 75.1725 & 79.7145 & 77.4435 \\
\hline GG & $\begin{array}{l}- \\
-\end{array}$ & $\begin{array}{l}- \\
-\end{array}$ & $\begin{array}{r}6.7895 \\
(0.6715)\end{array}$ & $\begin{array}{c}0.0217 \\
(0.1409)\end{array}$ & $\begin{array}{c}20.5814 \\
(131.756)\end{array}$ & 74.0367 & 77.4431 & 75.7399 \\
\hline
\end{tabular}

\section{Conclusions}

We present a new four-parameter model, called the transmuted generalized gamma (TGG) distribution, which extends the generalized gamma (GG) distribution and includes as special cases several distributions published in the literature. We provide some mathematical properties of the new distribution including expansions for the moments, generating function, mean devi-ations and Lorenz and Bonferroni curves. The maximum likelihood estimation of the model parameters is discussed and the observed information matrix is determined. An application to real data is performed in order to provide evidence that the TGG distribution can give a 

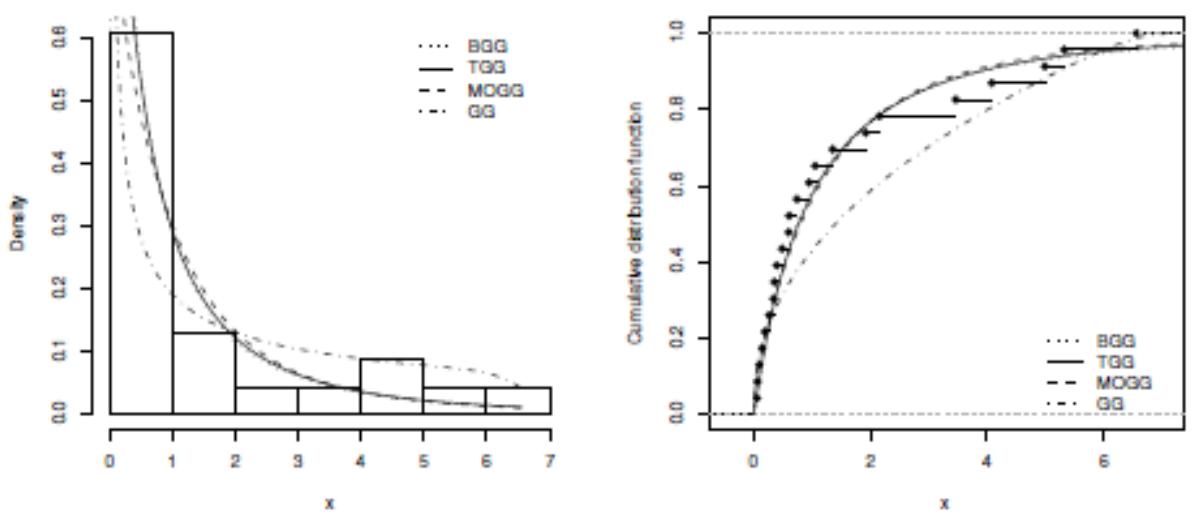

Figure 4: Histogram and estimated densities of the BGG, TGG, MOGG and GG models (left panel) and empirical cumulative function (right panel) of times between successive failures (in thousands of hours) of secondary reactor pumps

better fit than the GG distribution and some of its extensions. The results indicate that the new distribution outperforms some other extended models based on the GG distribution.

\section{Acknowledgments}

The authors are very grateful to the financial support from CAPES. We are grateful to the

Editor-in-Chief and an anonymous referee for the constructive comments. 


\section{Appendix}

The elements of the observed information matrix $J(\theta)$ for the parameters $(\lambda, a, v, p)$ are:

$$
\begin{aligned}
& J_{\lambda \lambda}=\sum_{i=0}^{n} \frac{y_{\lambda \lambda}\left(x_{i}\right)}{y\left(x_{i}\right)}, \quad J_{\lambda a}=-\sum_{i=0}^{n} \frac{y_{\lambda a}\left(x_{i}\right)}{y\left(x_{i}\right)}, \quad J_{\lambda \nu}=\sum_{i=0}^{n} \frac{y_{\lambda \nu}\left(x_{i}\right)}{y\left(x_{i}\right)}, \\
& J_{\lambda p}=-\sum_{i=0}^{n} \frac{y_{\lambda p}\left(x_{i}\right)}{y\left(x_{i}\right)}, \quad J_{a a}=\frac{n p \nu}{a^{2}}+\frac{p(p+1)}{a^{p+2}} \sum_{i=1}^{n} x_{i}^{p}-\sum_{i=1}^{n} \frac{y_{a a}\left(x_{i}\right)}{y\left(x_{i}\right)}, \\
& J_{a \nu}=-\frac{n p}{a}+\sum_{i=0}^{n} \frac{2 \lambda\left[y_{a \nu 1}\left(x_{i}\right)+y_{a \nu 2}\left(x_{i}\right)\right]}{y\left(x_{i}\right)}, \\
& J_{a p}=-\frac{n \nu}{a}-\sum_{i=0}^{n} \frac{x^{p}}{a^{p+1}}\left[1+p \log \left(\frac{x_{i}}{a}\right)\right]-\sum_{i=0}^{n} \frac{2 \lambda\left[y_{a p 1}\left(x_{i}\right)+y_{a p 2}\left(x_{i}\right)\right]}{y\left(x_{i}\right)} \text {, } \\
& J_{\nu \nu}=-n \psi(\nu)-\sum_{i=0}^{n} \frac{2 \lambda\left[y_{\nu \nu 1}\left(x_{i}\right)+y_{\nu \nu 2}\left(x_{i}\right)-y_{\nu \nu 3}\left(x_{i}\right)\right]}{y\left(x_{i}\right)}, \\
& J_{\nu p}=-n \log (a)+\sum_{i=0}^{n} \log \left(x_{i}\right)+\sum_{1=0}^{n} \frac{2 \lambda\left[y_{\nu p}\left(x_{i}\right)+y_{\nu p 2}\left(x_{i}\right)\right]}{y\left(x_{i}\right)}, \\
& J_{p p}=-\frac{n}{p^{2}}+\sum_{i=0}^{n}\left(\frac{x_{i}}{a}\right)^{p} \log ^{2}\left(\frac{x_{i}}{a}\right)-\sum_{1=0}^{n} \frac{2 \lambda\left[y_{\nu p p 1}\left(x_{i}\right)+y_{p p 2}\left(x_{i}\right)\right]}{y\left(x_{i}\right)} .
\end{aligned}
$$


Sadraque E.F. Lucena, Ana Herm' inia A. Silva, Gauss M. Cordeiro

The quantities y $\lambda \lambda(x i), y \lambda a(x i), y \lambda \nu(x i), y \lambda p(x i)$, yaa (xi), yav1 (xi ), yav2 (xi ), yap1 (xi ), yap2 (xi ), yvv1 (xi ), yvv2 (xi ), yvv3 (xi), yvp1 (xi ), yvp2 (xi), ypp1 (xi), ypp2 (xi) and y(xi) are given by

$$
\begin{aligned}
& y_{\lambda}\left(x_{i}\right)=\left\{\Gamma(\nu)-2 \gamma\left[\nu,\left(x_{i} / a\right)^{p}\right]\right\}^{2}, y_{\lambda a}\left(x_{i}\right)=2 \Gamma(\nu) \frac{\partial \gamma\left[\nu,\left(x_{i} / a\right)^{p}\right]}{\partial a}, \\
y_{\lambda \nu}\left(x_{i}\right)= & 2 \Gamma(\nu)\left\{\psi(\nu) \gamma\left[\nu,\left(x_{i} / a\right)^{p}\right]-\frac{\partial \gamma\left[\nu,\left(x_{i} / a\right)^{p}\right]}{\partial \nu}\right\}, \\
y_{\lambda p}\left(x_{i}\right)= & 2 \Gamma(\nu) \frac{\partial \gamma\left[\nu,\left(x_{i} / a\right)^{p}\right]}{\partial p}, \\
y_{a a}\left(x_{i}\right)= & 2 \lambda\left\{2 \lambda\left(\frac{\partial \gamma\left[\nu,\left(x_{i} / a\right)^{p}\right]}{\partial a}\right)^{2}+\frac{\partial^{2} \gamma\left[\nu,\left(x_{i} / a\right)^{p}\right]}{\partial a^{2}}\left[(1+\lambda) \Gamma(\nu)-2 \lambda \gamma\left[\nu,\left(x_{i} / a\right)^{p}\right]\right]\right\}, \\
y_{a \nu 1}\left(x_{i}\right)= & \frac{\partial^{2} \gamma\left[\nu,\left(x_{i} / a\right)^{p}\right]}{\partial \nu \partial a}\left[2 \lambda \gamma\left[\nu,\left(x_{i} / a\right)^{p}\right]-(1+\lambda) \Gamma(\nu)\right], \\
y_{a \nu 2}\left(x_{i}\right)= & \frac{\partial \gamma\left[\nu,\left(x_{i} / a\right)^{p}\right]}{\partial a}\left[(1+\lambda) \Gamma(\nu) \psi(\nu)-2 \lambda \frac{\partial \gamma\left[\nu,\left(x_{i} / a\right)^{p}\right]}{\partial \nu}\right], \\
y_{a p 1}\left(x_{i}\right)= & \frac{\partial^{2} \gamma\left[\nu,\left(x_{i} / a\right)^{p}\right]}{\partial p \partial a}\left[(1+\lambda) \Gamma(\nu)-2 \lambda \gamma\left[\nu,\left(x_{i} / a\right)^{p}\right]\right], \\
y_{a p 2}\left(x_{i}\right)= & 2 \lambda \frac{\partial \gamma\left[\nu,\left(x_{i} / a\right)^{p}\right]}{\partial p} \frac{\partial \gamma\left[\nu,\left(x_{i} / a\right)^{p}\right]}{\partial a}, \\
y_{\nu \nu 1}\left(x_{i}\right)= & 2 \lambda\left(\frac{\partial \gamma\left[\nu,\left(x_{i} / a\right)^{p}\right]}{\partial \nu}\right)^{2}+(1+\lambda) \Gamma(\nu) \frac{\partial^{2} \gamma\left[\nu,\left(x_{i} / a\right)^{p}\right]}{\partial \nu^{2}}, \\
y_{\nu \nu 2}\left(x_{i}\right)= & \gamma\left[\nu,\left(x_{i} / a\right)^{p}\right]\left[(1+\lambda) \Gamma(\nu)\left[\psi(\nu)^{2}-\psi^{\prime}(\nu)\right]-2 \lambda \frac{\partial^{2} \gamma\left[\nu,\left(x_{i} / a\right)^{p}\right]}{\partial \nu^{2}}\right], \\
y_{\nu \nu 3}\left(x_{i}\right)= & 2(1+\lambda) \Gamma(\nu) \psi(\nu) \frac{\partial \gamma\left[\nu,\left(x_{i} / a\right)^{p}\right]}{\partial \nu}+2 \lambda \psi^{\prime}(\nu) \gamma\left[\nu,\left(x_{i} / a\right)^{p}\right]^{2}, \\
y_{\nu p 1}\left(x_{i}\right)= & \frac{\partial^{2} \gamma\left[\nu,\left(x_{i} / a\right)^{p}\right]}{\partial p \partial \nu}\left[2 \lambda \gamma\left[\nu,\left(x_{i} / a\right)^{p}\right]-(1+\lambda) \Gamma(\nu)\right], \\
y_{\nu p 22}\left(x_{i}\right)= & \frac{\partial \gamma\left[\nu,\left(x_{i} / a\right)^{p}\right]}{\partial p}\left[(1+\lambda) \Gamma(\nu) \psi(\nu)-2 \lambda \frac{\partial \gamma\left[\nu,\left(x_{i} / a\right)^{p}\right]}{\partial \nu}\right], \\
y_{p p 1}\left(x_{i}\right)= & 2 \lambda\left(\frac{\partial \gamma\left[\nu,\left(x_{i} / a\right)^{p}\right]}{\partial p}\right)^{2}, \\
y_{p p 2}\left(x_{i}\right)= & \frac{\partial^{2} \gamma\left[\nu,\left(x_{i} / a\right)^{p}\right]}{\partial p^{2}}\left[(1+\lambda) \Gamma(\nu)-2 \lambda \gamma\left[\nu,\left(x_{i} / a\right)^{p}\right]\right], \\
y\left(x_{i}\right)= & \left\{(1+\lambda) \Gamma(\nu)-2 \lambda \gamma\left[\nu,\left(x_{i} / a\right)^{p}\right]\right\}^{2} . \\
&
\end{aligned}
$$




\section{References}

[1] Allenby, G. M., Leone, R. P. and Jen, L. (1999). A Dynamic Model of Purchase Timing with Application to Direct Marketing. Journal of the American Statistical Association 94,365-374.

[3] Aryal, G.R. and Tsokos, C.P. (2009).On the transmuted extreme value distribution with application. Nonlinear Analysis: Theory, Methods and Applications 71, 1401-1407.

[5] Aryal, G.R. and Tsokos, C.P. (2011). Transmuted Weibull distribution: a generalization of the Weibull probability distribution. European Journal of Pure and Applied Mathematics 4, 89-102.

[6]

[7] Aushor, S.K. and Eltehiwy, M.A. (2013). Transmuted Exponentiated Lomax distribution. Australian Journal of Basic and Applied Sciences 7, 658-667.

[9] Bowley, A. L. (1920). Elements of statistics. C. Scribner's sons, New York.

[11] Cordeiro, G.M., Castellares, F., Montenegro, L.C. and de Castro, M. (2012). The beta generalized gamma distribution. Statistics (Berlin) 46, 1-13.

[13] Cordeiro, G.M., and de Castro, M., 2011. A new family of generalized distributions. Journal of Statistical Computation and Simulation 81, 883-893.

[15]Cordeiro, G.M., Ortega, E.M.M. and Cunha, D.C.C. (2013).The Exponentiated Generalized Class of Distributions. Journal of Data Science 11, 1-27.

[16]

[17]Cordeiro, G.M., Ortega, E.M.M. and Silva, G.O. (2011).The exponentiated generalized gamma distribution with application to lifetime data. Journal of Statistical Computa- tion and Simulation 81, 827-842.

[18]

[19]Cox, C. (2008). The generalized F distribution: An umbrella for parametric survival analysis. Statistics in Medicine 27, 4301-4312.

[21]Cox, C., Chu, H., Schneider, M.F. and Muñoz, A. (2007). Parametric survival analysis and tax- onomy of hazard functions for the generalized gamma distribution. Statistics in Medicine 26, 4352-4374.

[23]Eugene, N., Lee, C. and Famoye, F., 2002. Beta-normal distribution and its applications. Communications in Statistics - Theory and Methods 31, 497-512. 
[24] Gupta, R.C., Gupta, P.L. and Gupta, R.D. (1998). Modeling Failure Time Data by Lehman Alternatives. Communications in Statistics - Theory and Methods 27, 887-904. [25]

[26]Khan, M.S. and King, R. (2013). Transmuted modified Weibull distribution: a generalization of the modified Weibull probability distribution. European Journal of Pure and Applied Mathematics 6, 66-88.

[28]Lawless, J. F. (1982). The Statistical Models and Methods for Lifetime Data. Wiley, New York.

[30]Moors, J. J. A. (1988). A quantile alternative for kurtosis. Journal of the royal statistical society (Series D) 37, 25-32.

[32] Ortega, E.M.M., Cordeiro, G.M., Pascoa, M.A.R. (2011). The Generalized Gamma Geometric Distribution. Journal of Statistical Theor and Applications 10, 433-454.

[34]Pascoa, M. A. R., Ortega, E. M. M. and Cordeiro, G. M. (2011). The Kumaraswamy generalized gamma distribution with application in survival analysis. Statistical Methodology 8, $411-433$

[36] Salman, S.M. and Prayoto, S. (1999). Total time on test plot analysis for mechanical components of the RSG-GAS reactor. Atom Indones 25, 81-90.

[38] Shaw, W. and Buckley, I. (2007). The Alchemy of Probability Distributions: Beyond GramCharlier Expansions, and a Skew-Kurtotic-Normal Distribution .From a Rank Transmutation Map. Research report.

[40] Stacy, E.W. (1962). A generalization of the gamma distribution. Annals of Mathematical Statistics 33, 1187-1192.

[41]

[42]Wright, E. M. (1935). The asymptotic expansion of the generalized Bessel function. Proceed-ings of the London Mathematical Society 38, 257-270.

[44] Yamaguchi, K. (1992). Accelerated Failure-Time Regression Models with a Regression Model of Surviving Fraction: An Application to the Analysis of 'Permanent Employment' in Japan. Journal of the American Statistical Association 87, 284-292.

Received December 12, 2013; accepted July 26, 2014. 
Sadraque E.F. Lucena

Universidade Federal de Pernambuco

Departamento de Estat_stica, Cidade Universit_aria

Recife/PE, 50740\{540, Brazil

Ana Herm'inia A. Silva

Universidade Federal de Pernambuco

Departamento de Estat_stica, Cidade Universit_aria

Recife/PE, 50740\{540, Brazil

Gauss M. Cordeiro

Universidade Federal de Pernambuco

Departamento de Estat_stica, Cidade Universit_aria

Recife/PE, 50740\{540, Brazil 